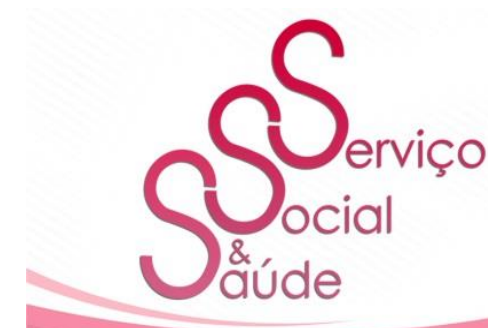

ISSN 2446-5992

(c) (1) (8)

doi: 10.20396/sss.v19i0.8665353

\title{
Desafíos para la Salud Mental en tiempos de crisis'
}

\author{
Challenges for Mental Health in times of crisis
}

Benedetto Saraceno $^{2}$

Buenos días a todos,

Agradezco a los organizadores del IX Taller UFPI ALASS de Formación Profesional y el Trabajo en Salud: los desafíos intersectoriales para el SUS, coordinado por la Profesora Joazeiro del Programa de Posgrado en Políticas Públicas de la Universidad Federal de Piauí, Brasil. Agradezco y me siento honrado por la invitación para hablar en esta importante sesión de Apertura. Gracias a todos por escuchar mi voz desde la lejana y fría ciudad de Ginebra.

La Declaración de Caracas fue adoptada por aclamación el 16 de noviembre de 1990 por doscientos participantes de once países latinoamericanos en la ciudad capital de Venezuela. Los principios postulados en la citada Declaración se refieren:

\footnotetext{
${ }^{1}$ Conferencia de Apertura de la Sesión Solemne del IX Taller UFPI ALASS, Universidad Federal de Piauí, Asociación Latina para el Análisis de Los Sistemas de Salud, ALASS, Barcelona, España, mediante el uso de tecnología remota, en 26.11.2020, con transmisión a través del Canal de Youtube ufpi tv, Universidade Federal de Piauí, Teresina, Brasil. Vídeo disponíble https://www.alass.org/pt/taller/ix-atelier-ufpi-alass-formacaoprofissional-e-trabalho-na-saude-a-intersetorialidade-desafios-para-o-sus/

${ }^{2}$ Prof. Dr. Benedetto Saraceno, Secretary General Lisbon Institute of Global Mental Health, CEDOC, New Medical School, Genebra. É médico psiquiatra, psicofarmacologista e sanitarista que trabalhou com Franco Basaglia e também na instituição de pesquisa de Milão, Instituto Mario Negri. Em 1990 organizou junto com Itzhak Levav, na época Diretor do Departamento de Saúde Mental da OPAS, a Conferência Regional para Reestruturação da Assistência Psiquiátrica no Continente, com a publicação da Declaração de Caracas, documento que tem norteado a Política de Saúde Mental no Brasil e em outros países das Américas. Ao longo da história tem sido um importante colaborador do Ministério da Saúde do Brasil desde 1992, tendo participado das II ${ }^{\mathrm{a}}$ e III ${ }^{\mathrm{a}}$ Conferências Nacionais de Saúde Mental e foi ex-Diretor do Departamento de Saúde Mental Álcool e Drogas da Organização Mundial de Saúde (OMS, WHO).
} 


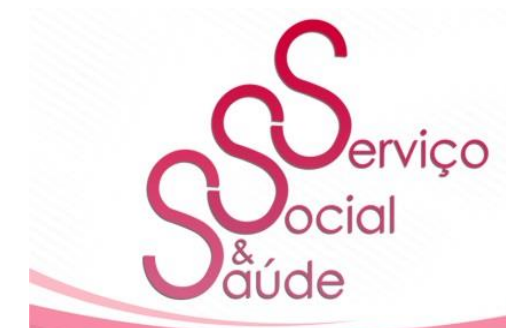

ISSN 2446-5992

(c) (1) (2)

doi: $10.20396 /$ sss.v19i0.8665353

1. A que el hospital psiquiátrico cesa de ser el componente central o único de la atención $\mathrm{y}$,

2. Establece los servicios en la comunidad como el eje principal para alcanzar una atención accesible, descentralizada, integral, participativa, continua y preventiva y radicalmente guiada por los derechos de los usuarios.

Hace 30 años los que pensaron y organizaron este evento (y tengo el privilegio de ser uno de ellos) estaban imaginando un sistema de salud mental no solamente más eficiente y efectivo sino también más cercano a las necesidades de los ciudadanos y de las comunidades. Caracas representó y sigue representando una hipótesis técnico-política de democratización de un segmento del sector salud, o sea el sistema de salud mental.

Nadie se imaginaba que treinta años después nos íbamos a enfrentar los desafíos de una pandemia global, duradera, mortal. Esta pandemia nos obliga a reformular nuestras hipótesis y quizás ponerlas al día. Esta pandemia ha desencadenado procesos de transformación de categorías que utilizamos diariamente y entre ellos nos obliga a tomar nueva conciencia sobre la dramática ausencia de democracia en los sistemas de salud, en general.

Hemos visto durante esta pandemia como el sistema de salud del rico Norte ha mostrado falta de cultura, organizaciones e infraestructuras capaces de operar incluso fuera del hospital. Muchas condiciones se han dejado agravar en el ámbito del hogar sin la capacidad de ofrecer ninguna intervención de mitigación que evite la agravación. Finalmente, muchas personas mayores alojadas en residencias especializadas murieron por la incapacidad de intervenir en estructuras que no eran "hospitales" y que se caracterizan por un fuerte componente de asistencia social no médica.

Entonces, el hospital fue la única respuesta junto con la adopción indispensable de medidas de distanciamiento social. Básicamente, entre la hospitalización urgente y el sentido cívico individual de las personas que respetan el confinamiento, no ha habido nada en el medio. 


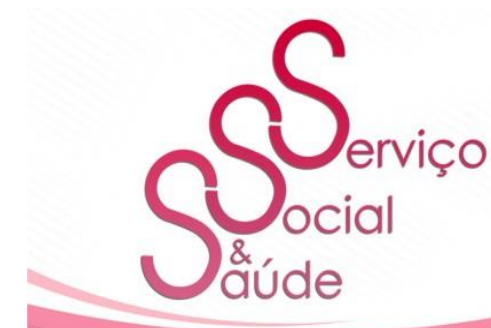

ISSN 2446-5992

(c) 10 (2)

doi: $10.20396 /$ sss.v19i0.8665353

La estrategia de algunos países ha mostrado su fragilidad e inequidad frente a la emergencia. No sorprende, teniendo en cuenta que estos países han venido deshaciéndose de la medicina comunitaria y de la salud en las comunidades y han desarrollado sólo los hospitales favoreciendo una privatización salvaje del sistema de salud pública.

Sin embargo, sabemos muy bien y desde muchos años atrás, que el modelo

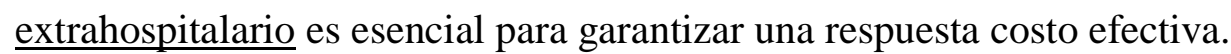

Una respuesta aguda no es automáticamente sinónimo de una cama de hospital. El desarrollo de un sistema de medicina en la comunidad y de una atención primaria "fuerte" debería haber sido, y mucho antes de la crisis pandémica, una elección prioritaria de los organismos responsables de la administración de la salud.

El fortalecimiento de la medicina local y de la Atención Primaria será uno de los grandes objetivos del "después". Esto quiere decir reconsiderar radicalmente las estrategias concretas que promueven la democracia en salud y el empoderamiento de los ciudadanos que demandan salud a los sistemas de salud pública.

Será muy útil a este propósito revisar la noción de "democracia profunda" (Deep democracy) introducida por el antropólogo Arjun Appadurai: la democracia profunda es la construcción de procesos democráticos desde abajo, es la experiencia de construir la ciudadanía de los más vulnerables a partir de los problemas concretos y diarios, para pasar de sobrevivir a vivir. Tenemos que aprender y planificar nuevas formas de salud local desde abajo

Un grupo de salubristas, economistas y profesionales de la salud estamos ahora, en Italia, trabajando en lo que llamamos "Casa de la Salud" que no es no identificable en un simple lugar donde se ofrecen intervenciones de salud sino un lugar en y para la Comunidad: lugar de recepción, de participación, lugar de superación de las desigualdades. En resumen, un lugar donde todos los actores de la convivencia (más allá de los profesionales de la salud) se encuentran planificando y gestionando juntos el bienestar presente y futuro de la comunidad misma. Entonces, la Casa della Salute pone a disposición de los habitantes intervenciones 


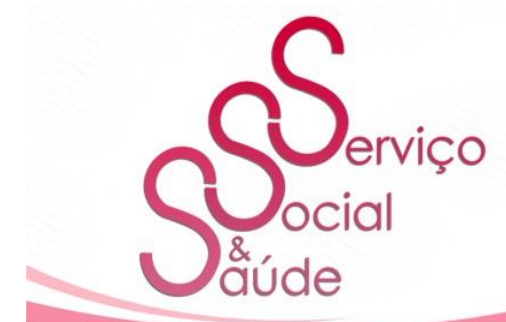

ISSN 2446-5992

(c) (1) (2)

doi: $10.20396 /$ sss.v19i0.8665353

4

capaces de mitigar y contrastar los efectos de los factores de riesgo no solo para la salud sino también para la dimensión más amplia que el antropólogo Arthur Kleinman llama "sufrimiento social".

Para lograr esta realización concreta del bienestar de la comunidad, será necesario finalmente poner a los determinantes sociales en el centro de la intervención de salud y no dejarlos como un fondo que se limite a aceptar intervenciones exclusivamente individuales y biomédicas. En realidad, existe la posibilidad de mitigar los efectos tanto de esos microdeterminantes sociales que contextualizan la vida de cada individuo como de los macrodeterminantes sociales que contextualizan la vida de cada comunidad.

Hoy es urgente y necesario liberar el tema de la enfermedad de una dimensión individual y biomédica exclusiva para, en cambio, mantenerlo coherente con la cuestión más general de una democratización radical de los sistemas de salud.

Es solamente dentro de este marco general de la democracia en salud que podemos mirar a los desafíos futuros para la Salud Mental.

Hay una impresionante continuidad en el tiempo porque a lo largo de los últimos 30 años no hubo los cambios que se podían esperar. Hablar de perspectiva futuras implica reconocer que los desafíos de hace treinta años siguen los mismos, a saber:

- La centralización de los recursos de salud mental en establecimientos separados del resto del sistema de salud impide una distribución racional de los recursos y concentra la mayoría de ellos en instituciones de baja calidad asistencial y alto riesgo de violaciones de los derechos humanos;

- La dificultad de la integración de la atención de salud mental en los servicios de atención primaria debido al: bajo número de personal capacitado para brindar atención de salud mental, a la ausencia generalizada de supervisión y a la errática disponibilidad de medicamentos psicotrópicos;

- La falta de organización y coordinación de los usuarios y de los familiares que siguen con opiniones muy diferentes (incluso contradictorias) acerca de los trastornos 


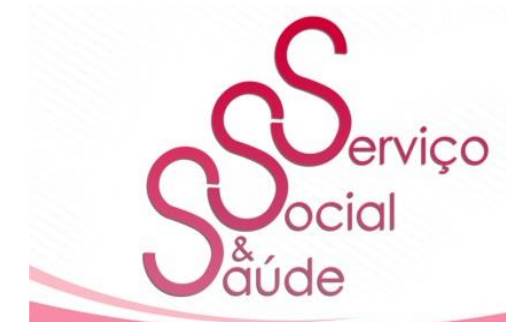

ISSN 2446-5992

(c) (1) (3)

doi: $10.20396 /$ sss.v19i0.8665353

mentales, de los tratamientos, los servicios, los derechos y de las medidas que hay que tomar para desarrollar la atención;

- La visión de los políticos, planificadores y salubristas que sigue equivocadamente basada en la opinión que los tratamientos de salud mental no son costo-efectivos y que la inversión en salud mental no es rentable desde el punto de vista de los resultados; y

- Una extendida hostilidad frente a la introducción de métodos evaluativos o, en todo caso, de cualquier intento de basar las intervenciones y las estrategias en la evidencia científica.

Además, es evidente que para lograr un cambio efectivo e innovador de la atención psiquiátrica hay que cambiar radicalmente la formación de los profesionales de la salud mental. La formación que se ofrece hoy en día en la mayoría de las universidades es exclusivamente clínica y, en muchos casos, no provee de los instrumentos "críticos" para leer críticamente la información psicofarmacológica, no capacita para navegar con inteligencia entre experiencia clínica y evidencia científica, para evaluar las intervenciones ni la capacidad para trabajar en equipo, y para considerar los derechos humanos como el componente fundamental de toda intervención psiquiátrica.

Es muy probable que la lección más importante de tantos años de intentos de reformas de la asistencia psiquiátrica, los aspectos de libertad, de ciudadanía, los derechos y la inclusión social permanecen como elementos irrenunciables de toda asistencia psiquiátrica y son constantes que superan las diferencias geográficas, culturales, sociales, económicas y lingüísticas. La Convención de las Naciones Unidas sobre Derechos de las Personas con Discapacidad sanciona esta universalidad de los derechos.

El futuro de la salud mental será cada vez más importante en las manos de tantos y tan diversos actores sociales: los profesionales de la salud mental, las organizaciones comunitarias, los activistas de los derechos humanos, los jueces y, evidentemente, los usuarios y los familiares que, tendrán que conquistar más poder y equilibrar siglos de opresión y de silencio. Gracias, Obrigado. 


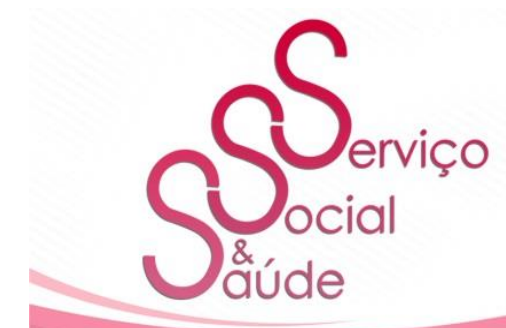

ISSN 2446-5992

(1) (0)

doi: $10.20396 /$ sss.v19i0.8665353

Recibido en noviembre de 2020 - Aprobado en noviembre de 2020

\section{REFERÊNCIAS}

APPADURAI, A. (Org.) Vida social das coisas, a - as mercadorias sob uma perspectiva cultural. Série Antropologia e Ciência Política, v. 41. Niteroi: Eduff. 2008.

KLEINMAN, A. Capítulo 1, Os significados pessoais e sociais da doença". The Illness Narratives: Suffering, Meaning, and the Human Condition. Basic Books, 1988, p. 31-55. OIT. ONU.OMS. Estrategia para la rehabilitación, la igualdad de oportunidades, la reducción de la pobreza y la integración social de las personas con discapacidad. Oficina Internacional del Trabajo, Organización de las Naciones Unidas para la Educación, la Ciencia y la Cultura y la Organización Mundial de la Salud. Organización Mundial de la Salud, 2005. Disponível

http://apps. who.int/iris/bitstream/handle/10665/43126/9243592386_spa.pdf;jsessionid=34036 ED4A76852854DC85E18018D6C4E?sequence=1

OPAS. OMS. Declaración de Caracas, Venezuela, 14 de noviembre de 1990, Adoptada por aclamación por la Conferencia Reestructuración De la Atención Psiquiátrica en América Latina Caracas, Venezuela, 11-14 de noviembre de 1990. Disponible en http://www.oas.org/dil/esp/Declaracion_de_Caracas.pdf 